\title{
POROUS MEDIA MICROSTRUCTURE RECONSTRUCTION USING PIXEL-BASED AND OBJECT-BASED SIMULATED ANNEALING - COMPARISON WITH OTHER RECONSTRUCTION METHODS
}

\begin{abstract}
A. N. Diógenes ${ }^{\mathrm{a}}$, ABSTRACT
L. O. E. dos Santos ${ }^{b}$,

C. P. Fernandes ${ }^{b}$,

A. C. Moreira ${ }^{b}$ and C. R. Apolloni ${ }^{\mathrm{c}}$

${ }^{a}$ Universidade Federal do Paraná, Departamento de Engenharia Mecânica, Bairro Jardim das Américas

CP. 19011, Curitiba, Paraná, Brasil andiogenes@ufpr.br

${ }^{\text {b }}$ Universidade Federal de Santa Catarina, Laboratório de Meios Porosos e Propriedades Termofísicas - LMPT CEP 88040-900 - C.P. 476 - Florianópolis, SC, Brasil

${ }^{\mathrm{c}}$ Universidade Estadual de Londrina, Campus

In this contribution the issue of the stochastic reconstruction of particulatemedia from 2D photomicrographic images is addressed with particular reference to pore space connectivity. The reconstruction of porous bodies in $2 \mathrm{D}$ or $3 \mathrm{D}$ space was achieved by using simulated annealing techniques. Two methods were proposed to reconstruct a well connected pore space. The first, named PSA (Pixel-based Simulated Annealing), a pixel-movement based, three constraints were found to be necessary for the successful reconstruction of well connected pore space: the two-point correlation function, the d3-4 distance transform distribution and the linealpath function for the pore phase. The second, named OSA (Object-based Simulated Annealing), only constrains the two-point correlation function. Following several researches which tried to reconstruct porous media using pixel-movement based simulated techniques, we propose a new parameter to add a microstructure descriptor, but we also propose a new technique, based in moving the microstructure grains (spheres) instead of the pixels. Both methods were applied to reconstruct reservoir rocks microstructures, and the 2D and 3D results were compared with microstructures reconstructed by truncated Gaussian methods. The PSA resulted in microstructures characterized by poor pore space connectivity, and by artificial patterns, while the OSA reconstructed microstructures with good pore space connectivity. These results indicate that the OSA method can reconstruct better microstructures than the present methods.
\end{abstract} Universitário - Cx. Postal 6001 CEP 86051-990 - Londrina - Paraná
Keywords: Simulated annealing, Porous media, Microstructure reconstruction, Reservoir rocks.

\section{NOMENCLATURE}

d Euclidean dimension

$\mathrm{d}_{34} \quad$ chamfer metric

E simulated annealing Energy

L image linear size

n number of spheres

$\mathrm{p} \quad$ simulated annealing probability

r sphere radius

$\mathrm{T}$ simulated annealing temperature

$\mathrm{t}$ iteration counter

\section{INTRODUCTION}

The quantitative relationships between the microstructure of porous media and their macroscopic properties are of great theoretical and practical relevance to many fields of technology, including oil recovery, hydrology, catalysis, adsorption and membrane separation. These relationships cannot be determined theoretically without considering the chaotic nature of the pore space of real porous media (Adler 1992; Torquato 2002; Sahimi 2003). Since variations in pore shape and pore space connectivity are intrinsic features of many porous media, a pore structure model capable of predicting the macroscopic properties must involve both geometric and topological descriptions of their complex microstructure. Recently, advanced techniques, such as X-ray computed microtomography (Spanne et al. 1994, Arns 2005) and magnetic resonance computed microtomography (Baldwin et al. 1996) have been introduced to obtain $3 \mathrm{D}$ volume images of pore space. However these techniques may be limited by their resolution, so an attractive alternative is the 3D stochastic reconstruction of porous solids from statistical information produced by analysis of 2D photomicrographs.

Provided that 2D photomicrographs of pore space are available, the main issue is to identify a set of microstructural descriptors that enable reliable reproduction of the pore space of the selected medium. The first reconstruction procedures were based on the thresholding of Gaussian random fields (TG). This approach, which has been widely studied (e.g. Quiblier 1984; Adler et al. 1990, Liang et al. 1998), is limited by the void fraction (porosity) and autocorrelation function of the medium under consideration. It has been evaluated by several 
researchers (Levitz 1998; Kainourgiakis et al. 2000; Hilfer 2000), all of whom found it incapable of reproducing the microstructure of particulatemedia, such as grain or sphere packs.

Another reconstruction methods preserving a different parameter, the pore size distribution, is the superposed spheres (SS). Dos Santos et al. (2002) developed the method for reconstruct a medium upholding this statistic. The method calculates the number of spheres in order to reconstruct a given porous media, saving it's the porosity. Each sphere superposes neighboring spheres according to a userdefined parameter. This method presents good results for connectivity, although it does not preserves the autocorrelation function.

A greater flexibility can be achieved by using the method of simulated annealing (SA) (Yeong and Torquato 1998a,b; Manwart et al. 2000; Talukdar and Torsaeter 2002; Talukdar et al. 2002a,b; Hamzehpour and Sahimi 2006, Arpat and Caers 2007, Capek et al. 2008). Using this technique of optimization, arbitrary microstructural geometrical descriptors may be imposed (within computational limits) as constraints on the reconstruction procedure. Several porous materials, including sandstone and chalk, have been successfully reconstructed by using a combination of various microstructural descriptors. Although this reconstruction procedure has been more successful, the resulting images do not always capture the longrange connectivity of pore space, namely for low porosity materials and particulatemedia as the reservoir rocks.

The porous microstructures geometries of reservoir rocks can present a highly complicated configuration and, consequently, several geometric and physical properties can be use to characterize these materials. Among these properties we can mention the porosity, the pore size distribution and the permeability, usually obtained in laboratory experiments. They are often expensive, time consuming and sample destructive. This is very important, since for the reservoir rocks, these experiments are accomplished in large pieces of rock called testimonies extracted from the soil.

Regard this scenario, the objective of this research is to present a technique for the porous media reconstruction in $3 \mathrm{D}$ using object-based and pixel-based simulated annealing methods. The main focus in the method is to preserve the connectivity and yet uphold desired geometrical parameters. The results will be compared with the TG method, which is the most used for this purpose and with some samples' X-ray microtomographies obtained using a SKYSCAN 1172 X-Ray microtomograph equipment located at the Petrobras Research Facility (CENPES). The comparison method will be measuring the quadratic error to the autocorrelation function, measured in microtomographies or images acquired with optical microscopy.
The paper is organized as follows: The chapter two explains the microstructure characterization and the reconstruction methods adopted. The chapter three shows the results when comparing the microstructure geometrical parameters. The chapter four presents some discussion about the results and the chapter five ends with conclusions.

\section{EXPERIMENTS}

This topic is divided into microstructure characterization and reconstruction.

\section{Microstructure characterization}

2D Characterization. One method to characterize the microstructure is to extract 2D information from the rock microstructure. The rocks' testimonies are sawed and a piece of it called plug is extracted. This plug is impregnated with resin, it is sawed again and a slice is grinded until it reaches $100 \mu \mathrm{m}$ thick. The microstructure images are obtained through optical microscopy and a sample result image is at Figure (1).

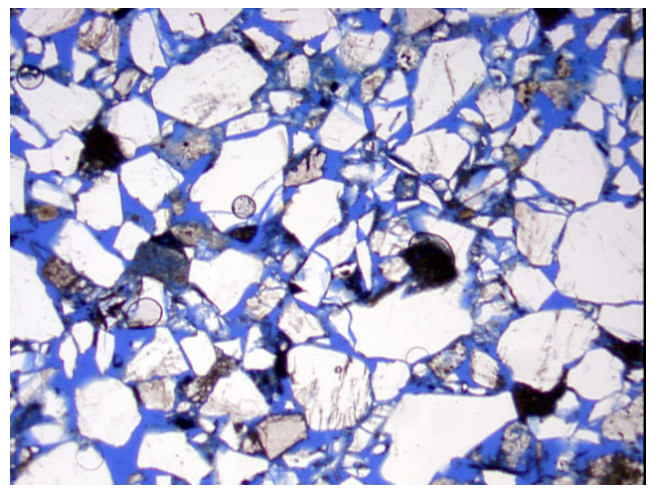

Figure 1. Rock sample image acquired by optical microscopy.

This image is segmented to find the porespace which is impregnated with the blue resin using the well-known HSI segmentation method (Gonzales \& Woods, 1992). An example is shown in Figure (2) with the porespace in white.

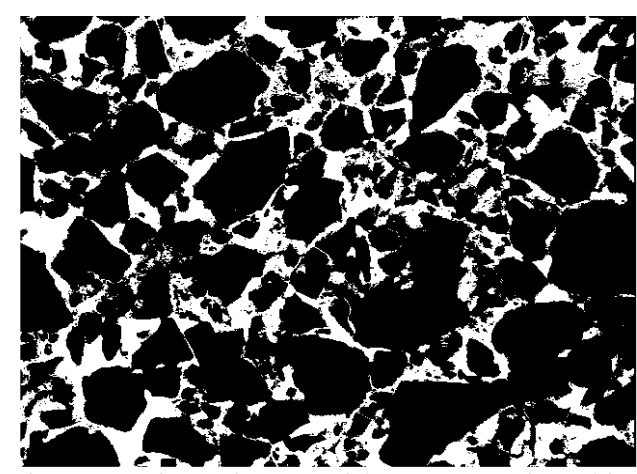

Figure 2. Binary image with porespace in White. 
From the binary image it is possible to measure the desired parameters, as frequential autocorrelation (Liang et al, 2000), chord length distribution and distance transform distribution (Adler, 1992). These parameters are used by the reconstruction methods to recreate the porespace in $3 \mathrm{D}$.

\section{Microstructure Reconstruction Methods}

The simulated annealing method was first developed by Cerny (1985) and Kirkpatrick et. al. (1983) to solve optimization problems (Aarts and Korst 1989). Stochastic reconstruction by the SA algorithm involves the gradual transformation of a high-energy configuration of pore and solid voxels into a minimum-energy configuration. The objective function ("energy" of the digitized system) This energy is usually defined as the sum of squared deviations between an arbitrary number of reference correlation functions, experimentally determined, and correlation functions calculated for 2D/3D replicas of a porous medium.

In order to use the simulated annealing algorithm, one has first to define a set of solutions, generally large, representing the solutions of an optimization problem. Then a neighborhood structure is defined. To find a good solution we move from a solution to one of its neighbors in accordance to the Metropolis probabilistic criterion shown by Eq. (1).

$$
p= \begin{cases}1 & \text { if } E_{t} \leq E_{t-1} \\ e^{\left(E_{t-1}-E_{t}\right) / T} & \text { if } E_{t}>E_{t-1}\end{cases}
$$

where $\mathrm{p}$ is the acceptance probability, Et is the energy for the iteration $\mathrm{t}$ and $e^{\left(E_{t-1}-E_{t}\right) / T}$ is the Boltzmann probability which decreases as the Temperature $\mathrm{T}$ decreases.

If the cost decreases then the solution is changed and the move is accepted. Otherwise, the move is accepted only with a probability depending on the cost increase and a control parameter called temperature. Classically, the probability to accept bad moves, i.e. moves with increase in terms of cost, is high at the beginning to allow the algorithm to escape from local minimum. This probability decreases in a progressive way by reducing the temperature. The method used to decrease the temperature is generally called cooling schedule. Some of the most common cooling schedules are the geometric and the adaptative (Ben-Ameur, 2004). This research adopted the geometric cooling schedule exposed at Eq. (2), descreasing the temperature after 1000 iterations.

$$
T_{t+1}=0,99 \cdot T_{t}
$$

Pixel-based method (PSA). This method preserves the well-known autocorrelation function, chord length distribution (Torquato, 2000) and $\mathrm{d}_{34}$ distance transform distribution. This last parameter is obtained by measuring the $\mathrm{d}_{34}$ distance transform distribution as in Chassery \& Montanvert (1991) and calculating each distance frequency along the entire image. Several authors (Hazlett, 1997, Yeong and Torquato, 1998a and Manwart et al., 2000) exposed that the autocorrelation function isn't enough for describing the porous media, so this research also adopted the above mentioned parameters expecting to conserve connectivity in the $3 \mathrm{D}$ reconstruction.

The process begins with a completely random image which has the same porosity as the porosity measured in the original images. Two randomly chosen pixels, one pore (1) and one solid (0), have their position exchanged and the desired parameters are calculated in the modified image. The acceptance rule presented above is used to admit or reject the exchange. The process goes on until a desired error or a time limit is achieved. The error target was $10^{-5}$ and the time limit was $24 \mathrm{~h}$ using a computer with a $2.2 \mathrm{GHz}$ frequency processor.

Object-based method (OSA). This method preserves only the autocorrelation function. It uses the grain size distribution as start point. For each sphere radius from up to down, it is calculated a number of spheres which fits inside the image using the Eq. (3):

$$
n(r)=\left(\frac{3}{4}\right)^{d-2} \cdot \frac{L^{d}}{\pi r^{d}}
$$

where $\mathrm{d}$ is the dimension ( 2 or 3 ), $\mathrm{L}$ is the image linear size and $r$ is the desired radius. This generates a spheres distribution which will be randomly disposed inside the porous media, allowing superposition.

After all the spheres are randomly disposed along the image and the process begins randomly selecting two spheres and exchanging their centers' positions. The autocorrelation is calculated in the modified image and the previous acceptance criterion is adopted. The process goes on the same way with the same error targets as the PSA method.

\section{RESULTS AND DISCUSSION}

The results obtained from both methods proposed here will be compared with Truncated Gaussian (Liang et al., 1998) and Superposed Spheres (dos Santos et al., 2002) results. The first method was also evaluated with reduced resolution. The resulting reconstructed images were generated with reduced resolution with factors of 2,3 and 4 , i.e., if the primary image had a $1 \mu \mathrm{m}$ resolution, the reconstructed image with amplification factor of 2 will have $2 \mu \mathrm{m}$ resolution.

\section{D results}


2D results are compared focusing the autocorrelation error. Figure (3) shows the binary primary image, referred in Table 1 as sample 1 . Figures (4) and (5) show the reconstructed images using the PSA and OSA methods, respectively.

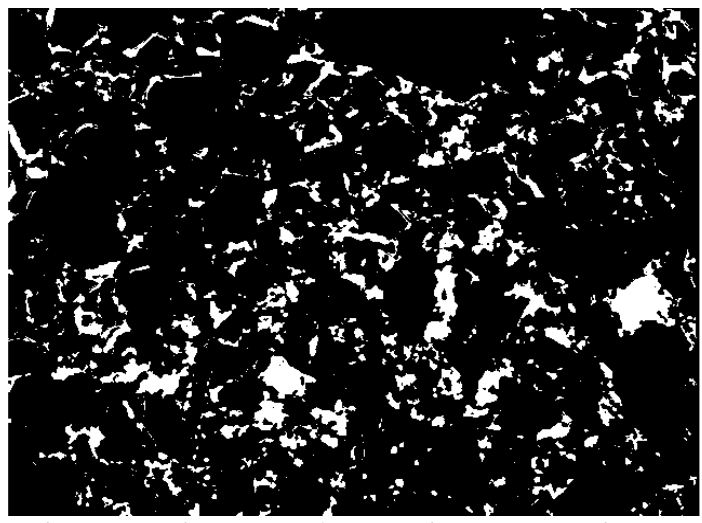

Figure 3. Primary sandstone microstructure image with $7.8 \mu \mathrm{m}$ resolution.

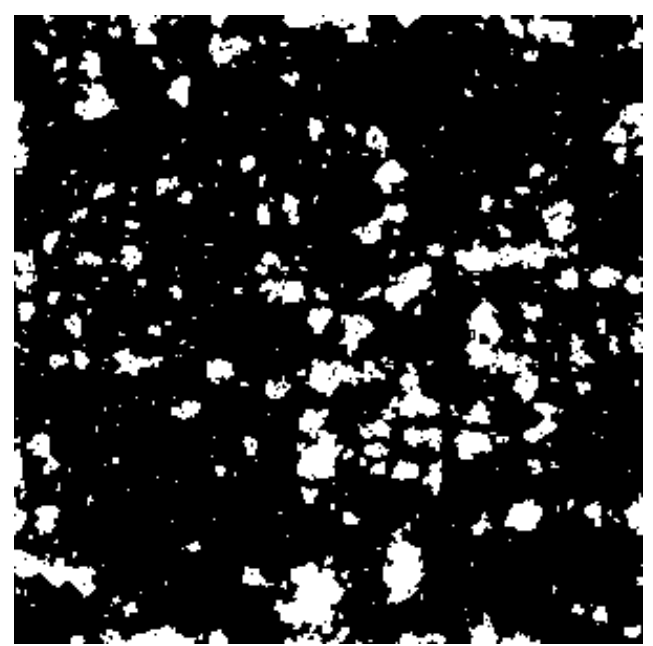

Figure 4. Sandstone microstructure image reconstructed using PSA with $7.8 \mu \mathrm{m}$ resolution.

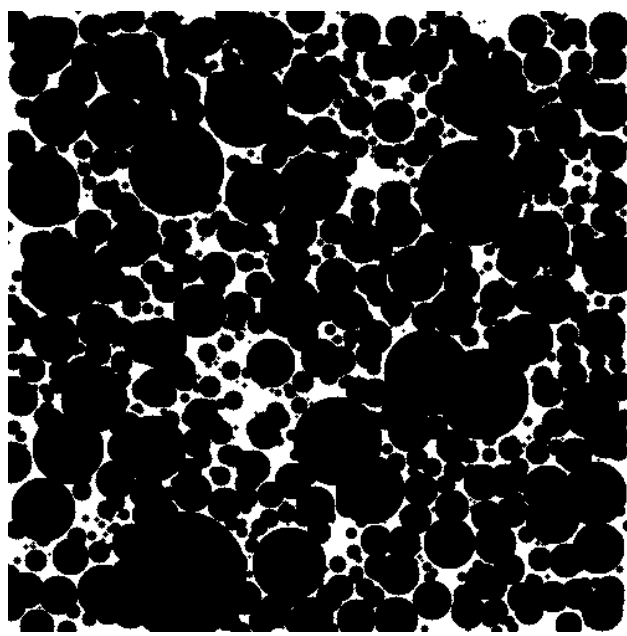

Figure 5. Sandstone microstructure image reconstructed using OSA with $7.8 \mu \mathrm{m}$ resolution.
It was analyzed the quadratic error for 8 samples. The data is presented in Table 1. The light gray shows the result with the least error. The dark gray shows the point where the TG method was better than both simulated annealing methods. The last column shows which method minimizes best the samples autocorrelation function quadratic error. This comparison was realized among the TG, PSA and OSA methods, since the TG method is the classical method for preserving autocorrelation function. The parenthesis for each TG cell indicates the factor that better reproduced the autocorrelation function.

Table 1 -Comparison of autocorrelation function quadratic error for 2D reconstruction methods

\begin{tabular}{|c|c|c|c|c|c|}
\cline { 3 - 6 } \multicolumn{2}{c|}{} & \multicolumn{2}{c|}{ Autocorrelation Function Quadratic Error } \\
\hline Samples & Porosity & PSA & OSA & TG-Best & Minimal \\
\hline $\mathbf{0 1}$ & $\mathbf{1 3 . 2 4 \%}$ & $1.85 \mathrm{E}-03$ & $4.75 \mathrm{E}-03$ & $1.80 \mathrm{E}-03(2)$ & TG-F2 \\
\hline $\mathbf{0 2}$ & $\mathbf{1 3 . 8 0 \%}$ & $7.74 \mathrm{E}-04$ & $6.08 \mathrm{E}-06$ & $4.10 \mathrm{E}-05(3)$ & OSA \\
\hline $\mathbf{0 3}$ & $\mathbf{1 5 . 6 6 \%}$ & $1.89 \mathrm{E}-03$ & $6.56 \mathrm{E}-05$ & $1.16 \mathrm{E}-04(4)$ & OSA \\
\hline $\mathbf{0 4}$ & $\mathbf{1 8 . 8 4 \%}$ & $5.98 \mathrm{E}-04$ & $1.84 \mathrm{E}-05$ & $1.74 \mathrm{E}-04(4)$ & OSA \\
\hline $\mathbf{0 5}$ & $\mathbf{1 9 . 5 0 \%}$ & $1.53 \mathrm{E}-02$ & $2.12 \mathrm{E}-05$ & $1.67 \mathrm{E}-03(4)$ & OSA \\
\hline $\mathbf{0 6}$ & $\mathbf{2 0 . 8 4 \%}$ & $1.28 \mathrm{E}-03$ & $7.85 \mathrm{E}-05$ & $4.11 \mathrm{E}-04(4)$ & OSA \\
\hline $\mathbf{0 7}$ & $\mathbf{2 3 . 9 3 \%}$ & $2.93 \mathrm{E}-03$ & $2.45 \mathrm{E}-05$ & $3.57 \mathrm{E}-04(2)$ & OSA \\
\hline $\mathbf{0 8}$ & $\mathbf{2 6 . 8 1 \%}$ & $2.99 \mathrm{E}-02$ & $2.82 \mathrm{E}-05$ & $4.27 \mathrm{E}-04(3)$ & OSA \\
\hline
\end{tabular}

As one can see, the OSA method showed better results than the other analyzed methods. These results show the method potential even within the presented porosity range.

\section{$3 D$ results}

The 3D results are compared focusing the error in the autocorrelation function. Table 2 shows that the OSA method had the least square error among the measured. The error was calculated considering the autocorrelation function for all $2 \mathrm{D}$ sections of the reconstructed $3 \mathrm{D}$ reconstruction. The best $3 \mathrm{D}$ reconstruction is shown in Figure (6b) in comparison with a microtomography sample Figure (6a).

The tomography measurements were accomplished with the following scan conditions: $60 \mathrm{kV}, 167 \mu \mathrm{A}$, Al filter $(1 \mathrm{~mm})$, each 5 frames averaged, $1475 \mathrm{~ms}$ integration time per frame, 180o total rotation angle with $0.45 \mathrm{o}$ step size of rotation angle. 815 images of sample slices were reconstructed with $5 \mu \mathrm{m}$ spatial resolution. The microtomography used for the experiments was a Skycan model 1072 located at the Petrobras research facility.

All the samples analyzed at this research were sandstones without clay. Due to confidentiality, the samples are referred as numbers. 


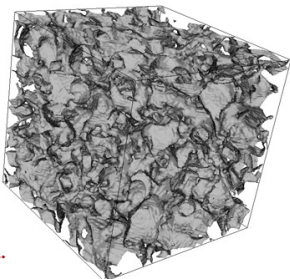

(a)

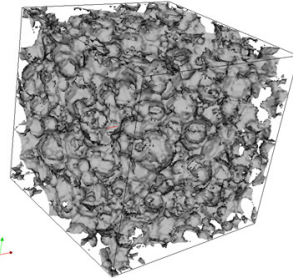

(b)
Figure 6. (a) Sample 09 microtomographic image with pore space in gray $\left(100^{3}\right.$ voxels) and (b) OSA

$3 \mathrm{D}$ reconstruction result with $100^{3}$ voxels. Both sample have $5.01 \mu \mathrm{m}$ spatial resolution.

Table 2 -Comparison of autocorrelation function quadratic errors for the $3 \mathrm{D}$ reconstruction methods researched in this work.

\begin{tabular}{|c|c|c|c|c|c|}
\cline { 3 - 6 } \multicolumn{2}{c}{} & \multicolumn{4}{|c|}{ Autocorrelation Function Quadratic Error } \\
\hline Sample & Porosity & PSA & OSA & TG-Best (AF) & Minimal \\
\hline $\mathbf{0 9}$ & $\mathbf{2 8 . 1 4 \%}$ & $3.54 \mathrm{E}-02$ & $5.11 \mathrm{E}-05$ & $6.01 \mathrm{E}-04(1)$ & OSA \\
\hline $\mathbf{1 0}$ & $\mathbf{1 4 . 6 8 \%}$ & & $7.00 \mathrm{E}-04$ & $3.52 \mathrm{E}-05(3)$ & $\begin{array}{c}\text { TG- } \\
\text { AF3 }\end{array}$ \\
\hline $\mathbf{1 1}$ & $\mathbf{0 9 . 3 0 \%}$ & & $1.78 \mathrm{E}-04$ & $7.54 \mathrm{E}-05(1)$ & TG-AF1 \\
\hline $\mathbf{1 2}$ & $\mathbf{1 4 . 6 4 \%}$ & & $1.90 \mathrm{E}-05$ & $1.10 \mathrm{E}-03(4)$ & OSA \\
\hline
\end{tabular}

Other important issue is to analyze the $2 \mathrm{D}$ sections of each 3D reconstruction. We analyzed the pore and grain size distributions using $\mathrm{d}_{34}$ (for 2D) and $\mathrm{d} 345$ (for 3D) chamfer metrics (Chassery \& Montanvert, 1991). This parameter, together with the connected porosity, shows how connected the pores are and how the porosity is distributed along the media. This will be very important to determine physical properties.

Table 3 exposes the pore and grain size distribution quadratic error for each $2 \mathrm{D}$ section of each reconstruction method. The error is calculated considering the microtomographic image for each sample.

Table $3-d_{34}$ pore and grain size distributions quadratic error for each reconstruction method.

\begin{tabular}{|c|c|c|c|c|}
\hline & \multicolumn{2}{|c|}{$\begin{array}{c}\mathbf{d}_{\mathbf{3 4}} \text { Pore Size Dist. } \\
\text { Error }\end{array}$} & \multicolumn{2}{c|}{$\begin{array}{c}\mathbf{d}_{\mathbf{3 4}} \text { Grain Size } \\
\text { Dist. Error }\end{array}$} \\
\hline Sample & OSA & SS & OSA & SS \\
\hline $\mathbf{0 9}$ & $6.12 \mathrm{E}-02$ & $7.86 \mathrm{E}-03$ & $5.29 \mathrm{E}-03$ & $1.30 \mathrm{E}-01$ \\
\hline $\mathbf{1 0}$ & $4.36 \mathrm{E}-03$ & $1.28 \mathrm{E}-02$ & $2.92 \mathrm{E}-02$ & $2.62 \mathrm{E}-03$ \\
\hline $\mathbf{1 1}$ & $3.31 \mathrm{E}-03$ & $2.68 \mathrm{E}-03$ & $8.39 \mathrm{E}-03$ & $2.64 \mathrm{E}-02$ \\
\hline $\mathbf{1 2}$ & $7.78 \mathrm{E}-03$ & $8.47 \mathrm{E}-02$ & $4.90 \mathrm{E}-03$ & $8.33 \mathrm{E}-03$ \\
\hline
\end{tabular}

Table 4 exposes the pore and grain size distribution for each 3D reconstruction method.

Table $4-\mathrm{d}_{345}$ pore and grain size distributions quadratic error for each reconstruction method.

\begin{tabular}{|c|c|c|c|c|}
\hline & \multicolumn{2}{|c|}{$\begin{array}{c}\mathbf{d}_{345} \text { Pore Size Dist. } \\
\text { Error }\end{array}$} & \multicolumn{2}{|c|}{$\begin{array}{c}\mathbf{d}_{345} \text { Grain Size Dist. } \\
\text { Error }\end{array}$} \\
\hline Sample & OSA & SS & OSA & SS \\
\hline 09 & $5.92 \mathrm{E}-02$ & $4.69 \mathrm{E}-02$ & $3.24 \mathrm{E}-03$ & $6.67 \mathrm{E}-03$ \\
\hline 10 & $1.86 \mathrm{E}-02$ & $3.11 \mathrm{E}-02$ & $1.11 \mathrm{E}-02$ & $1.22 \mathrm{E}-02$ \\
\hline 11 & $1.66 \mathrm{E}-02$ & $2.21 \mathrm{E}-01$ & $9.95 \mathrm{E}-03$ & $5.69 \mathrm{E}-03$ \\
\hline 12 & $3.71 \mathrm{E}-02$ & $1.71 \mathrm{E}-01$ & $5.38 \mathrm{E}-03$ & $7.58 \mathrm{E}-03$ \\
\hline
\end{tabular}

Table 5 shows another important parameter: the connected porosity. This parameter was calculated by eliminating the objects (pores) which don't percolate the media and by measuring the porosity after the elimination. The importance of this parameter comes from the necessity of the pore phase to percolate the media if ones intend to simulate a physical property like permeability. The Truncated Gaussian reconstructions were omitted since the method did not have good results in reproducing the connected porosity.

Table 5 -Connected porosity for each sample and reconstructions.

\begin{tabular}{|c|c|c|c|c|}
\hline Sample & Porosity & OSA & SS & Minimal \\
\hline $\mathbf{0 9}$ & $28.14 \%$ & $28.37 \%$ & $27.99 \%$ & SS \\
\hline $\mathbf{1 0}$ & $14.67 \%$ & $14.97 \%$ & $14.46 \%$ & SS \\
\hline $\mathbf{1 1}$ & $9.30 \%$ & $8.87 \%$ & $8.36 \%$ & OSA \\
\hline $\mathbf{1 2}$ & $14.64 \%$ & $15.33 \%$ & $12.09 \%$ & OSA \\
\hline
\end{tabular}

Table 6 shows the time consumption of each reconstruction method in reconstructing a microstructure with $80^{3}$ voxels using a $2.2 \mathrm{GHz}$ processor computer.

Table 6 -Time for each reconstruction method.

\begin{tabular}{|c|c|}
\hline Method & Time (s) \\
\hline PSA & 302,200 \\
\hline OSA & 1,100 \\
\hline TG & 0.1 \\
\hline SS & 4 \\
\hline
\end{tabular}

Table 7 shows results for the permeability determination for each reconstructed media and the $\mu$-CT. The permeability determination method is a lattice-Bolztmann method and the algorithm the used by dos Santos (2002). Again, the PSA and the SS methods are omitted due to bad results.

Table 7 -Permeability determination for each sample and reconstructed media.

\begin{tabular}{|c|c|c|c|}
\hline & \multicolumn{3}{|c|}{ Permeability (mD) } \\
\hline Sample & $\boldsymbol{\mu - C T}$ & OSA & TG-Best \\
\hline $\mathbf{0 9}$ & 4,223 & 1,779 & $1.829(1)$ \\
\hline $\mathbf{1 0}$ & 1,347 & 1,215 & $1(3)$ \\
\hline $\mathbf{1 1}$ & 226 & 577 & $1(1)$ \\
\hline $\mathbf{1 2}$ & 1,022 & 825 & $93(2)$ \\
\hline
\end{tabular}


As shown in Table 1, the OSA method was the one which better represented the autocorrelation function for 7 samples.

The PSA method is very slow and produces better reconstructions than the TG method for the same resolutions in most cases, but when the TG resolution is decreased, the microstructure is better represented by the latter method. However, while the TG reconstruction is performed in just a few seconds, the PSA takes several hours for 2D images and many days for 3D microstructures. This point is discouraging, but if someone develop faster parameters describing the microstructure this method can generate better reconstructions. We only had one $3 \mathrm{D}$ result for this method, as it is very time consuming. This result, however, didn't connect any porosity, so it was discarded as a 3D method for this research.

The OSA results are very encouraging. Even though the resulting image isn't visually similar to the primary one, the autocorrelation function error was effectively minimized, having the least quadratic error for 7 from 8 samples. The method is still very time-consuming. Indeed the $3 \mathrm{D}$ algorithm runs faster than the PSA, but still much slower than the TG or the SS. There is still a problem for correcting the grain size distribution measured in 2D images to fit a 3D grain size distribution. Several authors as DeHoff \& Rhines (1968) and Edwards (1980) presented solutions for these measurements and their methods will be analyzed in a future work for 2D grain size distribution correction. The 3D results were obtained measuring the microtomography pore size distribution for the analyzed samples.

Other point is that although some $3 \mathrm{D}$ mean quadratic errors were greater in the OSA, the summed quadratic errors were mainly smaller. This means that the OSA generated images are more homogeneous than the images generated with other methods. This is very important when simulating physical properties. The pore and grain size distributions errors were also smaller in the majority of the analyzed cases. This is also important, as this parameter is not considered during the optimization stage, but even so, it is indirectly optimized during the process.

The TG method didn't present good results, as in most cases, it demands a resolution reduction for reducing the autocorrelation error and even so, it had no better results than the OSA or the SS methods.

The SS method was very good for connecting the porosity, but it isn't designed to preserve the autocorrelation function, so it produced poorly results and wasn't even compared to the other methods. This method yet has another advantage, which is being very fast, if compared to the OSA and PSA methods.

The OSA method tended to have better permeability determination results than the other methods. This wasn't true for the sample 09, which had greater porosity.

\section{CONCLUSIONS}

The PSA method are not able to reconstruct connected porous media in $3 \mathrm{D}$, although it generates images with small autocorrelation quadratic errors. This shows that the autocorrelation function and the chord distribution functions aren't enough to guarantee the microstructure 3D connectivity.

The OSA method achieved the objective the PSA hasn't. It reconstructs microstructures with reasonable connectivity preserving only the autocorrelation function. This happened because the way the object movement is realized. Instead of moving isolated voxels, the method moves objects, and this improves the final connectivity. Even without considering the $\mathrm{d} 34$ pore and grain size distributions errors during the optimization stage, these parameters were mainly preserved. This was confirmed by comparing the pore size distribution with SS reconstructed media.

The permeability determination results were also encouraging. The OSA method tended to reproduce better the permeability than the other methods.

The time is still a barrier, as both methods are very time consuming. Even more if compared with the SS method, which offer similar results when the connectivity is compared. The next step in this research is to determine electric parameters in all these $3 \mathrm{D}$ microstructure reconstructions and in the microtomographic images and compare the results.

\section{ACKNOWLEDGEMENTS}

The authors acknowledge the Agência Nacional do Petróleo - ANP for funding this this research and the the Petrobras Research Facility (CENPES) for providing the microtomographies used in this research.

\section{REFERENCES}

Aarts, E., Korst, J., 1989, Simulated Annealing and Boltzmann Machines: A Stochastic Approach to Combinatorial Optimization and Neural Computing. Wiley

Adler, P. M., Jacquin, C. G., and Quiblier, J. A., 1990. Flow in simulated porous media. International Journal of Multiphase Flow, Vol. 16, No 4, pp. 691712

Adler, P. M,. 1992, Porous Media: Geometry and Transports, Butterworth, Heinemann

Arpat, G. B. and Caers, J., 2007, Conditional simulation with Patterns, Mathematical Geology, Vol. 39, No. 2, pp. 542-547.

Baldwin, C. A., Sederman, A. J., Mantle, M. D., Alexander, P., and Gladden, L. F., 1996, Determination and characterization of the structure of a pore space from 3D volume images, Journal of 
Colloid Interfaces Science, Vol. 181, No 1, pp. 7992.

Ben-Ameur, W., 2004, Computing the Initial Temperature of Simulated Annealing, Computational Optimization and Applications, Vol. 29, pp. 369-385.

Čapek, P. Hejtmánek, V. Brabec, L. Zikánová A. and Kočiř́ík M., 2008, Stochastic Reconstruction of Particulate Media Using Simulated Annealing: Improving Pore Connectivity, Transport in Porous Media, Vol 76, No 2, pp. 179-198.

Cerny, V., 1985, A thermodynamical approach to the traveling salesman problem: An efficient simulated algorithm, Journal of Optimization Theory and Applications, Vol. 45, pp. 41-51.

Chassery, J.M., Montanvert, A., 1991, Géométrie discrete en analyse d'images, Editions Hermès

DeHoff, R.T. and Rhines, F., 1968, Quantitative Microscopy, Mc Graw-Hill.

Dos Santos, L. O. E., Philippi, P. C., Fernandes, C. P., and Gaspari, H. C., 2002, Reconstrução tridimensional de microestruturas porosas com o método das esferas sobrepostas, in: Proceedings of the ENCIT 2002, Caxambu - MG, Brazil - Paper CIT02-0449

Edwards, S. F. and Wilkinson, D. R., 1980, The deduction of the probability distribution of sphere sizes in a random assembly from measurements on a cross-section through the assembly, Journal of Physics D: Applied Physics, Vol. 13, pp. 209-211.

Gonzales, R., and Woods, R., 1992, Digital Image Processing, Prentice Hall.

Hamzehpour, H., and Sahimi, M., 2006, Development of optimal models of porous media by combining static and dynamic data: The porosity distribution. Physical Review. E 74, 026308.

Hazlett, R. D., 1997, .Statistical Characterization and Stochastic Modeling of Pore Networks in Relation to Fluid Flow, Mathematical Geology, Vol. 29, No 6, pp. 467-478.

Hilfer, R., 2000, Local porosity theory and stochastic reconstruction for porous media, In: Mecke, K., Stoyan, D. (eds.) Statistical Physics and Spatial Statistics, Vol. 254 of Lecture Notes in Physics, pp. 203-241. Springer-Verlag.

Ioannidis, M. A., Kwiecien, M. J., and Chatzis, I., 1996, Statistical analysis of the porous microstructure as a method for estimating reservoir permeability, Journal of Petroleum Science Engineering, Vol. 16, pp. 251-261.

Kainourgiakis, M. E., Kikkinides, E. S., Steriotis, T. A., Stubos, A. K., Tzevelekos, K. P., and Kanellopoulos, N. K., 2000, Structural and transport properties of alumina porous membranes from process-based and statistical reconstruction techniques, Journal of Colloid Interfaces Science, Vol. 231, 158-167.

Kirkpatrick, S., Gelatt, C. D. and Vecchi, M. P., 1983, Optimization by simulated annealing, Science, Vol. 220, pp. 671-680.
Levitz, P., 1998, Off-lattice reconstruction of porous media: critical evaluation, geometrical confinement and molecular transport, Advances Colloid and Interface Science, Vols. 76-77, pp. 71106.

Liang, Z. R., Fernandes, C. P., Magnani, F. S., and Philippi, P. C., 1998, A reconstruction technique for three-dimensional porous media using image analysis and Fourier Transform, Journal of Petroleum Science, Vol. 21, pp. 273-283.

Liang, Z., Ioannidis, M. A., and Chatzis, I., 2000, Permeability and electrical conductivity of porous media from $3 \mathrm{D}$ stochastic replicas of the microstructure, Chemical Engineering Science, Vol. 55, pp. 5247-5262.

Manwart, C., Torquato, S., and Hilfer, R., 2000, Stochastic reconstruction of sandstones, Physical Review E, Vol. 62, No 41, pp. 365-371.

Quiblier, J. A., 1984, A new three-dimensional modeling technique for studying porous media, Journal of Colloid Interface Science, Vol. 98, pp. 84102.

Sahimi, M., 2003, Heterogeneous Materials I \& II, Springer-Verlag

Spanne, P., Thovert, J. F., Jacquin, C. J., Lindquist, W. B., Jones, K. W., and Adler, P. M., 1994, Synchrotron computed microtomography of porous media: topology and transports. Physical Review Letters, Vol. 73, No 14, pp. 2001-2004.

Talukdar, M. S., and Torsaeter, O., 2002, Reconstruction of chalk pore networks from 2D backscatter electron micrographs using a simulated annealing technique, Journal of Petroleum Science Engineering, Vol. 33, pp. 265-282.

Talukdar, M. S., Torsaeter, O., Ioannidis, M. A., and Howard, J. J. 2002a, Stochastic reconstruction, 3D characterization and network modeling of chalk. Journal of Petroleum Science Engineering, Vol. 35 No 1-2, pp. 1-21.

Talukdar, M. S., Torsaeter, O., Ioannidis, M. A., and Howard, J. J., 2002b, Stochastic reconstruction of chalk from 2D images, Transport in Porous Media Vol. 48, pp. 101-123.

Torquato, S., 2002, Random Heterogeneous Materials: Microstructure and Macroscopic Properties. Springer-Verlag.

Yeong, C. L. Y. and Torquato, S., 1998a, Reconstructing random media, Physical Review E Vol. 58, No 1, pp. 495-506.

Yeong, C. L. Y. and Torquato, S., 1998b, Reconstructing random media II - Three-dimensional media from two dimensional cuts, Physical Review E Vol 58, No 1, pp. 224-233.

Received: June 09, 2009

Revised: July 09, 2009

Accepted: August 09, 2009 